\title{
The structure of $\mathrm{Al}(111)-\mathrm{K}-(\sqrt{3} \times \sqrt{3}) \mathrm{R} 30^{\circ}$ determined by LEED: stable and metastable adsorption sites
}

\author{
C. Stampfl ${ }^{\text {a }}$, J. Burchhardt ${ }^{b}$, M. Nielsen ${ }^{b}$, D.L. Adams ${ }^{\text {b }}$, M. Scheffler ${ }^{a}$, H. Over ${ }^{\text {a }}$ \\ and $\mathrm{W}$. Moritz ${ }^{\mathrm{c}}$ \\ ${ }^{a}$ Fritz-Haber Institut der Max-Planck-Gesellschaft, Faradayweg 4-6, D-14195 Berlin 33, Germany \\ ${ }^{b}$ Institute of Physics, Aarhus University, DK-8000 Aarhus C, Denmark \\ ' Institut für Kristallographie und Mineralogie, Universität München, D-8000 München 2, Germany
}

Received 1 September 1992; accepted for publication 7 October 1992

It is found that the adsorption of potassium on $\mathrm{Al}(111)$ at $90 \mathrm{~K}$ and at $300 \mathrm{~K}$ both result in a $(\sqrt{3} \times \sqrt{3}) \mathrm{R} 30^{\circ}$ structure. Through a detailed LEED analysis it is revealed that at $300 \mathrm{~K}$ the adatoms occupy substitutional sites and at $90 \mathrm{~K}$ the adatoms occupy on-top sites; both geometries have hitherto been considered as very unusual. The relationship between bond length and coordination is discussed with respect to the present results, and with respect to other quantitative studies of alkali-metal/metal adsorption systems.

Recently alkali-metal adsorption on metal surfaces has attracted much attention due to the controversial views concerning the nature of the bond (ref. [1], and references therein). It is being found that the adsorption system which was widely believed to be the most simple, based on earlier experimental results [2-5], is, from very recent studies [2,6-16], revealing itself as being much more complex. Moreover, to date, most of the unexpected and unusual behaviour has occurred on the close-packed surfaces - the ones previously thought to be the least complicated with occupation of maximally coordinated sites without reconstruction of the substrate.

The results of the present LEED study show that $\mathrm{K}$ adsorption on $\mathrm{Al}(111)$ at room temperature leads to a reconstruction of the substrate such that $\mathrm{K}$ atoms occupy substitutional sites and adsorption of $\mathrm{K}$ at low temperature results in occupation of on-top sites on a rumpled substrate. The possibility of this unexpected geometry was predicted by recent density-functional theory (DFT) studies [14] and confirmed by a detailed LEED analysis [15]. An irreversible transformation from the on-top, metastable structure to the substitutional structure occurs upon warming to room temperature.

In this paper we extend the previous analysis [15] and discuss our results, which correspond to a high-coverage situation, analysing the structural phase transition of the adlayer. $(\Theta=1 / 3$ is, for $K$ on $\mathrm{Al}(111)$ at room temperature, the saturation coverage.) Also, the present results, together with results of other studies on alkali-metal/metal systems, are discussed with respect to bonding and coordination.

A detailed LEED analysis was performed for cach of the $(\sqrt{3} \times \sqrt{3}) \mathrm{R} 30^{\circ}$ structures for four symmetry-inequivalent integral-order and four symmetry-inequivalent fractional-order beams obtained for normal incidence. The fully dynamical LEED intensity scheme [17] includes the possibility of automatic, simultaneous refinement of the structural parameters, the real part of the muffin-tin zero, and the cffective Debye temperature [18]. Details concerning the experimental procedure and theoretical calculations are given elsewhere [15]. 
For the structure formed at $300 \mathrm{~K}$ the analysis showed that the $\mathrm{K}$ atoms reside in quasi-substitutional sites formed by the ejection of $1 / 3$ of a monolayer of $\mathrm{Al}$ atoms. The $\mathrm{K}$ atoms are situated at a vertical distance of $2.16 \pm 0.03 \AA$ above the first layer of $\mathrm{Al}$ atoms, corresponding to a nearest-neighbour $\mathrm{K}-\mathrm{Al}$ distance of $3.58 \AA$. Assuming a hard-sphere metallic radius $r_{\mathrm{Al}}=1.43 \AA$ yields an effective radius $r_{K}=2.15 \AA$, which lies between the covalent $(2.03 \AA)$ and metallic $(2.38 \AA)$ radii. The first $\mathrm{Al}-\mathrm{Al}$ interlayer spacing was found to be contracted by $2 \% \pm 1 \%$ with respect to the bulk value, whereas the second $\mathrm{Al}-\mathrm{Al}$ interlayer spacing is equal to the bulk value within the error of the determination. A comparison of the experimental intensity curves with those calculated for the determined geometry is shown in fig. 1a, where very good agreement is evident. A sketch of the surface structure is shown in fig. 2a.

For adsorption of $\mathrm{K}$ at $90 \mathrm{~K}$ the analysis revealed that in this case the $\mathrm{K}$ atoms are located in on-top positions with a rumpling of the first layer of $\mathrm{Al}$ atoms, such that the $\mathrm{Al}$ atoms directly beneath the $K$ atoms are displaced towards the bulk by $0.25 \pm 0.04 \AA$. The vertical distance from the $K$ atoms to the uppermost atoms of the rumpled layer was found to be $2.98 \pm 0.05 \AA$, giving a $\mathrm{K}-\mathrm{Al}$ nearest-neighbour distance of 3.23 $\AA$. The vertical distances of the atoms in the rumpled layer to the second layer of $\mathrm{Al}$ atoms were found to be $2.46 \AA$ and $2.21 \pm 0.05 \AA$. The inward displacement of $1 / 3$ of the $\mathrm{Al}$ atoms in the first layer was found to be accompanied by a small outward lateral shift of $0.05 \AA$ of the nearest-neighbour $\mathrm{Al}$ atoms in the second layer. In this case an effective radius $r_{K}=1.80 \AA$ is obtained, assuming the same hard-sphere metallic radius. The effective radius lies between the ionic $(1.33 \AA)$ and covalent $(2.03 \AA)$ radii. Fig. $1 b$ shows a comparison of the experimental and calculated intensities where good agreement can be seen. The on-top structure described above is illustrated in fig. $2 b$.

It is interesting to compare other quantitatively determined structures of different alkalimetal/metal adsorption systems (see table 1), in particular, bond length and coordination. In fig. 3 the determined values of the effective radius ob-

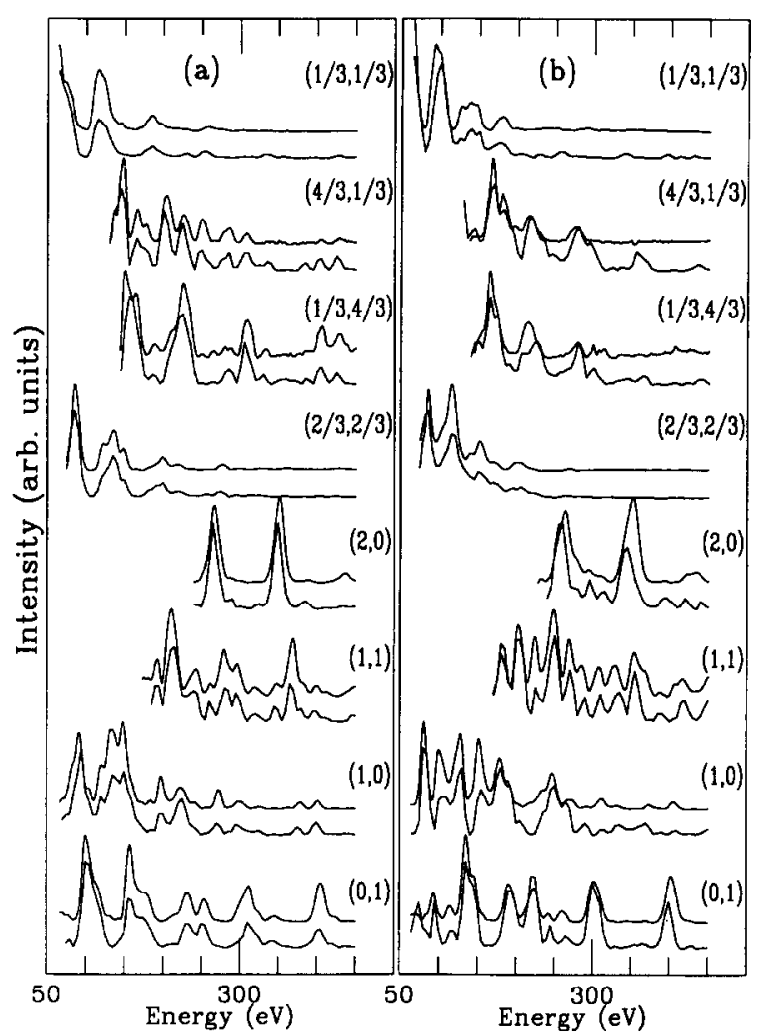

Fig. 1. Comparison of the experimental and calculated intensity-energy curves where the theoretical curves appear as the lower curve for each beam: (a) for the determined substitutional site as described in the text for adsorption of $\mathrm{K}$ at 300 $\mathrm{K}$ and (b) for the determined on-top structure as described in the text for adsorption of $\mathrm{K}$ at $90 \mathrm{~K}$.

tained in the present work, represented by full circles, are plotted against coordination number. The results of DFT calculations [14] are also included in the diagram, the points appearing as stars connected by solid lines; for $\mathrm{K} / \mathrm{Al}(111)$ there are two curves, the upper corresponding to a $(\sqrt{3} \times \sqrt{3})$ R $30^{\circ}$ structure $(\Theta=1 / 3)$ the lower to a $(2 \times 2)(\Theta=1 / 4)$ structure. Note, that the lower coverage structures have a smaller bond length. This is consistent with the idea that at lower coverage the bonding is more ionic [19]. The two values determined in the present analysis are joined by a dashed line plotted in such a way as to facilitate comparison with the DFT results. Also shown on the plot is a curve, with the points 
shown as open circles, obtained using the empirical relationship given by Kittel [20].

It can be seen from fig. 3 that on going from one-fold to three-fold coordination approximately the same increase in bond length $(\sim 0.3 \AA)$ is exhibited for both the DFT results and those of Kittel. The same increase is observed in the Cs/Ru(0001) system (see table 1), although here the coverages are different $(\Theta=0.25$ for the ontop one-fold coordinated site and $\Theta=0.33$ for the hcp-hollow three-fold coordinated site).

The effective radius of the adatom depends on its charge, therefore any change in ionicity should be reflected in a change of the adsorbate-substrate bond length. An increase in bond length with coordination is a general rule for ionic and covalent bonding, reflecting the idea that the bond length increases and bond strength decreases. The observed increase mentioned above is in accordance with these ideas. Indeed, nearly all of the determined values, as seen from table 1 , having the one-fold coordinated on-top site are such that the effective radius is closest to the ionic radius; and the effective radii for the adatoms occupying the three-fold coordinated hollow sites have an effective radius closest to the covalent ones.

From fig. 3 it is apparent that the obtained values of the change in bond length between coordinations 3 and 6 is different for the DFT calculations and those obtained using the relationship of Kittel. The values being approximately 0.04 (average) and $0.2 \AA$, respectively. As discussed in ref. [14] this (together with their obtained different gains in binding energy (between coordinations 1 and 3 , and 3 and 6)) indicates different bonding mechanisms for "normal" adsorption and "substitutional" adsorption. The present results allow a quantitative comparison to be made since for the same coverage, species and

Table 1

Determined structural parameters $(\AA)$ for the alkali-metal/metal systems listed

\begin{tabular}{|c|c|c|c|c|c|c|c|c|c|}
\hline System & Structure & Site & $C$ & Method & $\begin{array}{l}d_{\mathrm{nn}} \\
(\AA)\end{array}$ & $\begin{array}{l}\text { eff-r } \\
(\AA)\end{array}$ & $\begin{array}{l}\text { ion-r } \\
(\AA)\end{array}$ & $\begin{array}{l}\text { met-r } \\
(\AA)\end{array}$ & $\begin{array}{l}\text { cov-r } \\
(\AA)\end{array}$ \\
\hline $\mathrm{Na} / \mathrm{Ni}(100)[3]$ & $c(2 \times 2)$ & holl. & 4 & LEED & 2.84 & 1.59 & 0.97 & 1.91 & 1.54 \\
\hline $\mathrm{Na} / \mathrm{Al}(100)[5]$ & $c(2 \times 2)$ & holl. & 4 & LEED & 2.86 & 1.43 & 0.97 & 1.91 & 1.54 \\
\hline $\mathrm{Na} / \mathrm{Al}(111)[12]$ & $\sqrt{3} \times \sqrt{3}$ & sub. & 6 & SEXAFS & 3.31 & 1.88 & 0.97 & 1.91 & 1.54 \\
\hline $\mathrm{K} / \mathrm{Co}(1010)[11]$ & $c(2 \times 2)$ & holl. & 4 & LEED & 3.12 & 1.87 & 1.33 & 2.38 & 2.03 \\
\hline $\mathrm{K} / \mathrm{Ru}(0001)[13]$ & $p(2 \times 2)$ & f-h. & 3 & LEED & 3.25 & 1.94 & 1.33 & 2.38 & 2.03 \\
\hline $\mathrm{K} / \operatorname{Ru}(0001)[13]$ & $\sqrt{3} \times \sqrt{3}$ & h-h. & 3 & LEED & 3.29 & 1.98 & 1.33 & 2.38 & 2.03 \\
\hline $\mathrm{K} / \mathrm{Ni}(111)[9]$ & $\mathrm{p}(2 \times 2)$ & top & 1 & LEED & 2.82 & 1.57 & 1.33 & 2.38 & 2.03 \\
\hline $\mathrm{K} / \mathrm{Al}(111)$ & $\sqrt{3} \times \sqrt{3}$ & top & 1 & LEED & 3.23 & 1.80 & 1.33 & 2.38 & 2.03 \\
\hline $\mathrm{K} / \mathrm{Al}(111)$ & $\sqrt{3} \times \sqrt{3}$ & sub. & 6 & LEED & 3.58 & 2.15 & 1.33 & 2.38 & 2.03 \\
\hline $\mathrm{Rb} / \mathrm{Al}(111)[8]$ & $\sqrt{3} \times \sqrt{3}$ & top & 1 & SXW & 3.13 & 1.70 & 1.48 & 2.55 & 2.16 \\
\hline Cs/Rh(100) [10] & $c(4 \times 2)$ & holl. & 4 & LEED & 3.44 & 2.10 & 1.67 & 2.73 & 2.35 \\
\hline $\mathrm{Cs} / \mathrm{Ru}(0001)[7]$ & $\sqrt{3} \times \sqrt{3}$ & h-h. & 3 & LEED & 3.52 & 2.20 & 1.67 & 2.73 & 2.35 \\
\hline $\mathrm{Cs} / \mathrm{Ru}(0001)[7]$ & $p(2 \times 2)$ & top & 1 & LEED & 3.25 & 1.90 & 1.67 & 2.73 & 2.35 \\
\hline $\mathrm{Cs} / \mathrm{Cu}(111)[6]$ & $p(2 \times 2)$ & top & 1 & LEED & 3.01 & 1.73 & 1.67 & 2.73 & 2.35 \\
\hline $\mathrm{Cs} / \mathrm{Ag}(111)$ [19] & $\Theta=0.15$ & - & - & SEXAFS & 3.20 & 1.76 & 1.67 & 2.73 & 2.35 \\
\hline Cs/Ag(111) [19] & $\Theta=0.3$ & - & - & SEXAFS & 3.50 & 2.06 & 1.67 & 2.73 & 2.35 \\
\hline
\end{tabular}

For brevity " $\sqrt{3} \times \sqrt{3}$ " denotes $(\sqrt{3} \times \sqrt{3})$ R30 and "holl.", f-h.", "h-h." and"sub." are abbreviations for hollow, fcc hollow, hcp hollow and substitutional, respectively. Similarly, "C", “ $d_{n n}$ ", "eff-r", "ion-r", "met-r" and "cov-r" represent coordination number, determined nearest-neighbour distance, determined effective radius, and the ionic, metallic and covalent radii of the adsorbate, respectively. 
surface unit cell, two differently coordinated adsorption sites are obtained. From fig. 3 it can be seen that the present results agree well with the DFT results where the change in bond length between the one-fold and six-fold coordinated sites are 0.35 and $0.32 \AA$, for the present analysis and those of DFT, respectively.

The results presented here for the LEED analysis can be understood in that for the on-top site there is a large substrate relaxation and the $\mathbf{K}-\mathrm{K}$ repulsion can be efficiently screened. This site is metastable and on warming, the activation barrier can be overcome and an irreversible transition takes place to the substitutional site. The key factors making the substitutional site possible being the low vacancy formation energy and the even more reduced alkali-alkali repulsion [14].

For alkali-metal adsorption on the cubic (100) surfaces appearing in table 1 occupation of the maximally coordinated four-fold hollow sites were found, in accordance with earlier expectations,

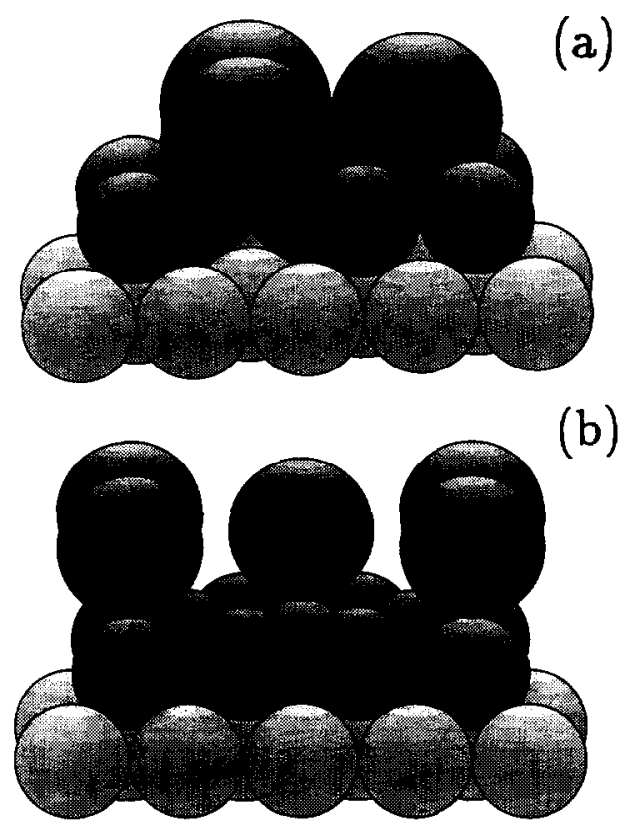

Fig. 2. Illustration of the atomic geometry where the $K$ atoms are shown as large dark-grey spheres and the $\mathrm{Al}$ atoms appear as smaller spheres. (a) The substitutional site and (b) the on-top site where the obtained rumpling of the first Al layer, as described in the text, is not apparent in the figure.

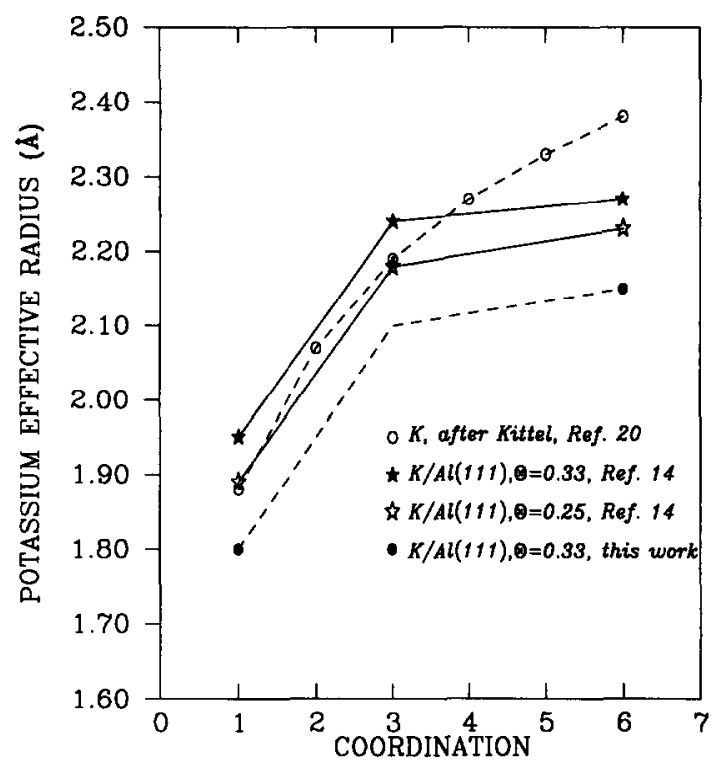

Fig. 3. Plot of the potassium effective radius versus coordination. The values of the present results (joined by a dashed line) are shown as full circles. The results of the DFT calculations [14] are shown as stars and are connected by a solid line. The open circles connected by a dashed line are obtained using the relationship given by Kittel [20].

where the effective radii in these cases lie between the ionic and covalent radii.

\section{Acknowledgements}

The authors wish to acknowledge useful discussions with Jesper Andersen, Jochen Haase, Jörg Neugebauer and Renee Diehl. Support of this work by the Danish Natural Science Research Council and Center for Surface Reactivity is gratefully acknowledged.

\section{References}

[1] M. Scheffler, Ch. Droste, A. Fleszar, F. Máca, G. Wachutka and G. Barzel, Physica B 172 (1991) 143.

[2] For a recent review, see: N.D. Lang, in: Physics and Chemistry of Alkali Metal Adsorption, Eds. H.P. Bonzel, A.M. Bradshaw and G. Ertl (Elsevier, Amsterdam, 1989).

[3] S. Andersson and J. Pendry, Solid State Commun. 16 (1975) 563. 
[4] J. Demuth, D. Jepsen and P. Marcus, J. Plys. C 8 (1975) L25.

[5] B. Hutchins, T. Rhodin and J. Demuth, Surf. Sci. 54 (1976) 419.

[6] S. Lindgren, L. Walldén, J. Rundgren, P. Westrin and J. Neve, Phys. Rev. B 28 (1983) 6707.

[7] H. Over, H. Bludau, M. Skottke-Klein, G. Ertl, W. Moritz and C. Campbell, Phys. Rev. B 45 (1992) 8638.

[8] M. Kerkar, D. Fisher, D.P. Woodruff, R. Jones, R. Diehl, C. McConville and B. Cowie, J. Vac. Sci. Technol. A 10 (1992) 2148;

M. Kerkar, D. Fisher, D.P. Woodruff, R. Jones, R. Diehl and B. Cowie, Phys. Rev. Lett. 68 (1992) 3204; Surf. Sci. 278 (1992) 246.

[9] D. Fisher, S. Chandavarkar, I.R. Collins, R. Diehl, P. Kaukasoina and M. Lindroos, Phys. Rev. Lett, 68 (1992) 2786.

[10] G. von Eggeling, G. Schmidt, G. Besold, L. Hammer, K. Heinz and K. Müller, Surf. Sci. 221 (1989) 11.

[11] C.J. Barnes, P. Hu, M. Lindroos and D.A. King, Surf. Sci. $251 / 252$ (1991) 561 .

[12] A. Schmalz, S. Aminprooz, L. Becker, J. Haase, J.
Neugebauer, M. Scheffer, D.R. Batchelor, D.L. Adams and E. Bøgh, Phys. Rev. Lett. 67 (1991) 2163.

[13] M. Gierer, H. Bludau, T. Hertel, H. Over, W. Moritz and G. Ertl, Surf. Sci. Lett. 279 (1992) L170.

[14] J. Neugebauer and M. Scheffier, Phys. Rev. B 46 (1992) 16067.

[15] C. Stampfl, M. Scheffler, H. Over, J, Burchhardt, M. Nielsen, D.L. Adams and W. Moritz, Phys. Rev. Lett. 69 (1992) 1532 .

[16] J.N. Andersen, E. Lundgren, R. Nyholm and M. Qvarford, Phys. Rev. B 46 (1992) 12784.

[17] W. Moritz, J. Phys. C 17 (1983) 353.

[18] G. Kleinle, W. Moritz and G. Ertl, Surf. Sci. 238 (1990) 119.

[19] G.M. Lamble, R.A. Brooks, D.A. King and D. Norman, Phys. Rev. Lett. 61 (1988) 1112.

[20] C. Kittel, Introduction to Solid State Physics (Wiley, New York, 1986) p. 77, tables 9 and 10. (The quantity shown in fig. 3 is $\Delta_{\mathrm{N}}+$ metallic adsorbate radius. This is an empirical relationship which assumes the nature of the adsorbate-substrate bond is the same irrespective of the coordination.) 